\title{
Enzootic bovine leucosis in cows on farms in Almaty and Turkestan regions of Kazakhstan
}

\author{
Assem Ibazhanova ${ }^{1 *}$, Ajdar Namet $^{2}$, Banu Nurgazy $^{1}$, Zhuldyzaj Kenzhebekova $^{1}$, Gabdolla \\ Shmanov ${ }^{1}$ \\ ${ }^{1}$ Kazakh National Agrarian University, 050000 Almaty, Kazakhstan \\ ${ }^{2}$ Kazakh Research Veterinary Institute, 050016 Almaty, Kazakhstan
}

\begin{abstract}
The objective of this study was to evaluate pathomorphological and histopathological characteristics of internal organ tissues and skeletal muscles of Black-Motley cows with bovine laeukemia necropsied between 2013 and 2017. Pathomorphological and histopathological assessment of enzootic bovine leukosis was performed. The viral presence was proved by haematological study, enzyme-linked immunosorbent assay and agar-gel immunodiffusion. There was the pathomorphological manifestation of an immunosuppressive condition in the cattle, as well as leukemic cell accumulations in the brain, kidneys, duodenum, spleen, lymph nodes, cardiac and skeletal muscles. Our study is considered to bear significance concerning the description of microstructural changes involving lymph node trabeculae and myocardial fibres from leukemic cows. This investigation, therefore, provides new knowledge on histopathological features of enzootic bovine leucosis, which potentially may bring new insights into the disease mechanisms.
\end{abstract}

\section{Introduction}

The problem of bovine laeukemia virus (BLV) is one of the most important in modern biology, ecology, human and veterinary medicine. BLV is reported to overcome the interspecific barrier [1]. It can reproduce in cultured human cells. When BLV is in contact with human cell culture, there is a destabilization of the cell genome with the formation of syncytium and activation of the tax gene, causing tumour transformation of the genome, which poses a risk of development of cancer $[2,3]$.

BLV burden remains high worldwide [4], so investigation of pathomorphological and histopathological changes in internal organs of BLV-infected cattle is considered actual. A pathoanatomical investigation of the visceral organs from slaughtered cows may contribute to timely diagnosis and prevention of the disease spread.

Enzootic bovine leucosis (EBL) is one of the common lymphatic proliferative diseases causing agricultural losses, largely due to the negative impact on the gene pool of highproductive cattle. Both young and adult cattle of any breed suffer from laeukemia, but it is more frequently diagnosed in animals of five to eight years of age [5].

* Corresponding author: assemibazhanova@yahoo.com 
Examination of structural changes in the internal organs enables to detect EBL earlier and perform preventive measures. Our research was aimed at studying pathomorphological and histopathological conditions of tissues of internal organs and skeletal muscles from BLV-positive cows.

\section{Materials and Methods}

The research was conducted at the Department of Biological Safety at Kazakh National Agrarian University (Kazakhstan, Almaty) from 2013 to 2017. Tissues submitted for evaluation were internal organs and skeletal muscles from carcasses of 13 adult BlackMotley cows delivered from private farms in Almaty and Turkestan regions of Kazakhstan for determination of a cause of death. Specimens collected from slaughtered cows were positive for BLV antibodies and antigens according to haematological study, enzymelinked immunosorbent assay (ELISA) and agar-gel immunodiffusion (AGID).

The animals were necropsied, and the details were recorded. Pronounced degenerative changes were recorded using a digital camera.

For histological examination, $0.5 \times 0.5 \times 1.0 \mathrm{~cm}$ samples were collected from various parts of the brain, lungs, kidneys, heart, spleen, liver, lymph nodes, gastrointestinal tract, and skeletal muscles. The materials were fixed in $10 \%$ solution of neutral formalin, then dehydrated in $60 \%, 70 \%, 80 \%, 90 \%, 96.1 \%$, and $96.2 \%$ ethanol. The tissue sections were embedded into paraffin and paraffin-celloidine, and 5-15 $\mu \mathrm{m}$ sections were obtained using a cryostat. The slices were stained with hematoxylin-eosin, by Van Gieson and RomanovskyGiemsa methods for microscopic observation by means of Leica DMLS microscope.

\section{Results and Discussion}

Macroscopic changes in the studied carcasses were similar: they were skinny; the mucous membranes of the mouth cavity and eyes were bright yellow, with a pink tint. In the peritoneal cavity, we found 0.5-1.0 litres of a transparent orange fluid, that is common to lymphoma [6].

Lymph nodes were enlarged, friable, oval, light yellow in colour. The greatest increase was observed in the subclavian and inguinal lymph nodes (Fig. 1). They were of an oily consistency, elastic, juicy, glistening, with yellowish section surface.

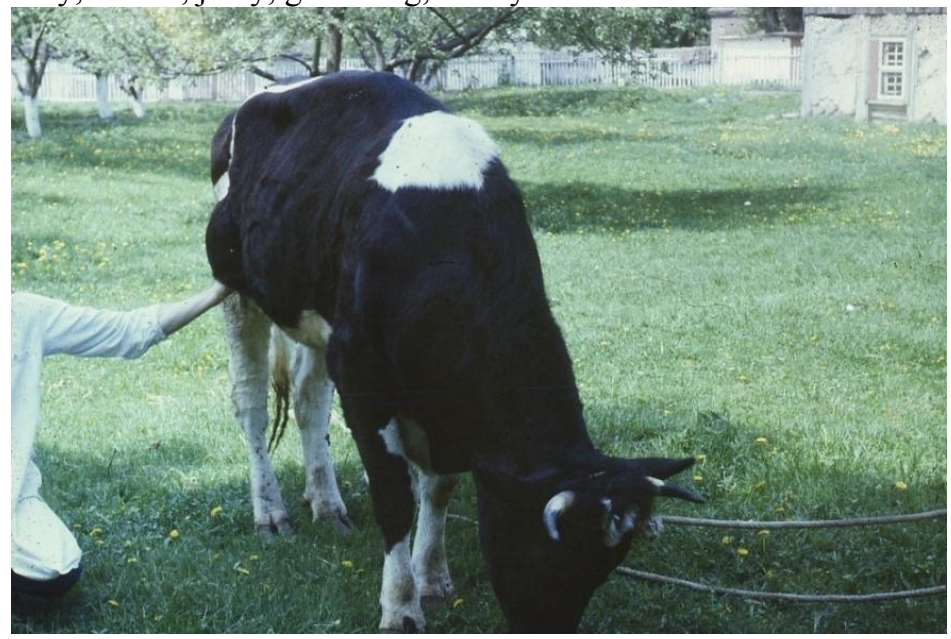

Fig. 1. Enlarged subclavian and inguinal lymph nodes 
Follicular hyperplasia in the spleen was observed. It is suggested to be associated with immune dysfunction preceding the development of the tumour [7]. At the same time, the spleen was not enlarged (due to reduced area of red pulp), it was light grey, the cut surface was raspberry-red, coarse-grained, the pulp protruded through the capsule, the latter was not tense (Fig. 2).

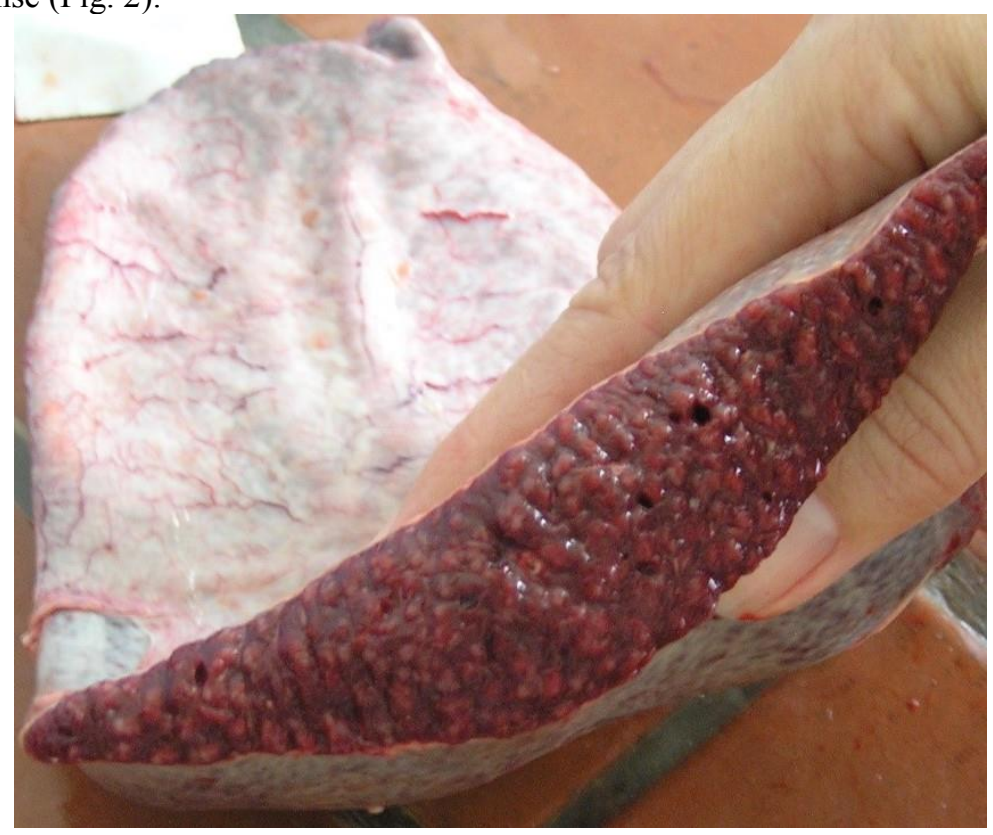

Fig. 2. Follicular hyperplasia of the spleen in the cows

Immunosuppressive effects of BLV include oncogenesis [8]. According to the published literature, neoplastic transformations in the heart induced by BLV affect mainly the right atrium but may localize throughout the myocardium and extend to the pericardium $[9,10]$. In this study, we recorded cardiac neoplasms notably in the right atrium. Myocardium was stiff, of inhomogeneous light pink colour, with grey inclusions. The structure of the fibres was pronounced. The epicardium was pale, moist, and glistening. The endocardium was light grey, smooth, and moist (Fig. 3). 


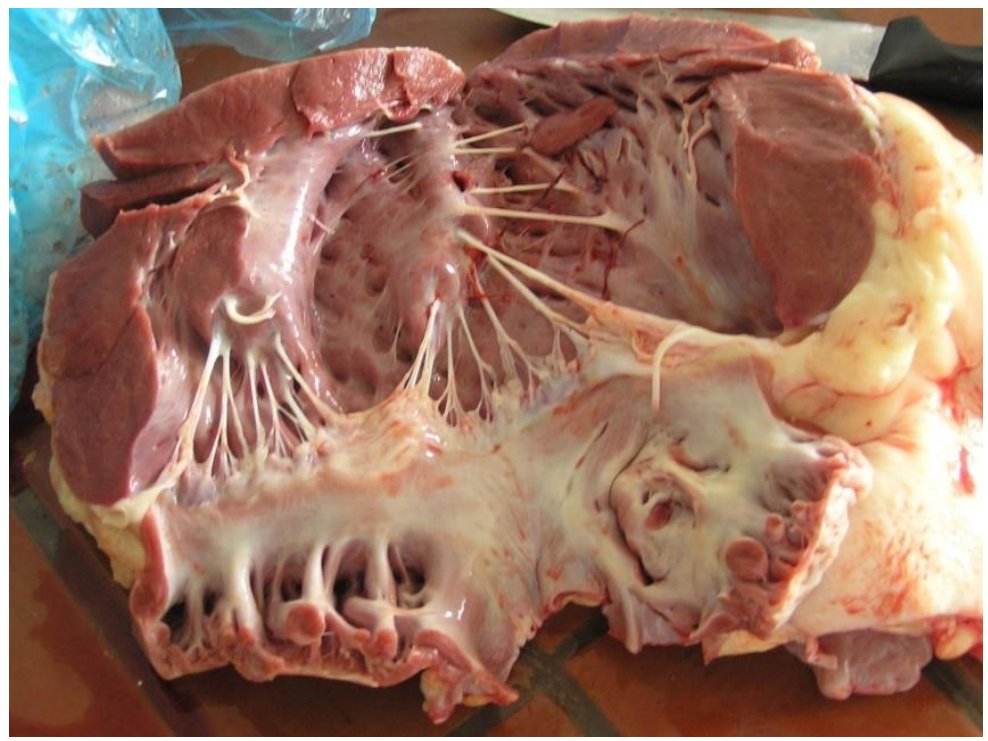

Fig. 3. Diffuse lesions in cardiac tissue that were assumed to be lymphoid proliferate

The kidneys were moderately enlarged and bumpy, grey-brown, of dense consistency, with the diffuse demarcation between the renal cortex and medulla. The capsule was transparent, moist, greyish, easily removable, and not tense.

The lungs were unenlarged, light pink, elastic, and had a distinct multi-lobed structure.

The liver capsule was tense, but the organ was not enlarged, its edges remained sharp, but lobar segmentation was poorly discernible. The capsule and the section surface were dark red. In the liver parenchyma, small fuzzy brown-grey lesions were noted (Fig. 4).

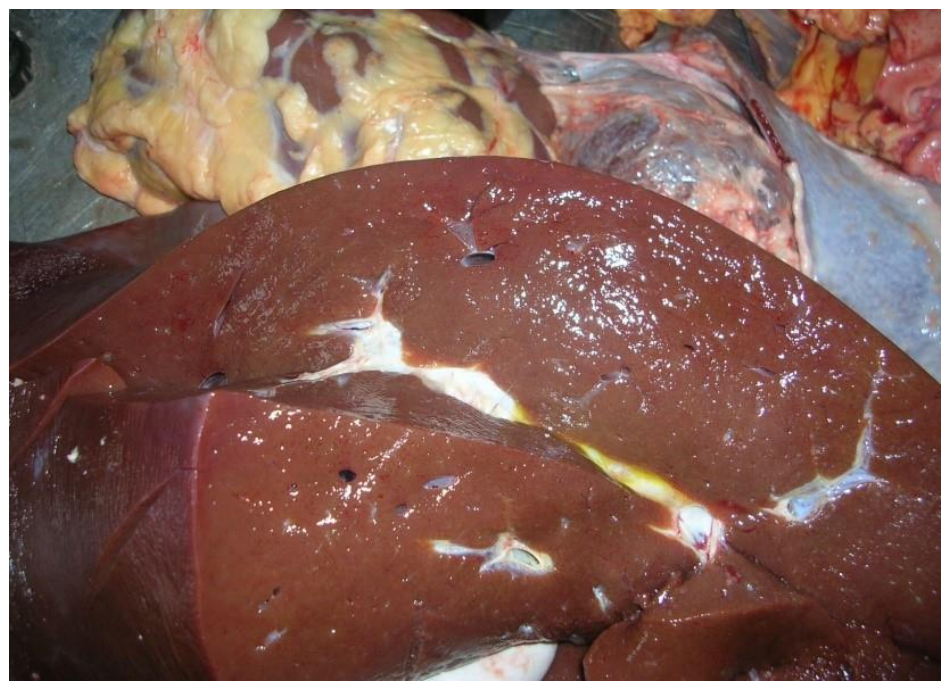

Fig. 4. Diffuse lesions in the liver of the cows

The duodenum was light grey, with locally thickened wall, and filled with undigested greenish matter. A single case of erosion and ulcer formation has been recorded. In dairy cattle, this may be associated, inter alia, with gastric lymphosarcoma [11]. 
In the small intestine, hypertrophied lymphoid follicles were observed. The mucous membrane was light grey, moist, and the intestinal wall thickness was homogeneous. The mucosa of the large intestine was unthickened and light grey in colour.

In the muscles of the back, pelvis, and shoulder girdle, light grey foci were noted, presumably associated with the development of leukaemia [12].

There were no lesions in the hard and soft shells of the brain. The softshell was inflamed, and the blood vessels were half-filled with blood. No visible pathological changes were present in the cerebral gyri and sulci. Small amounts of clear fluid were found in the ventricles of the brain.

Histologically, lymph nodes characterized by enlargement of lymphoid follicles. A similar phenomenon is described in histopathological findings from a research work performed by McCune and Yuuka [13]. The loose lymph node cortex was infiltrated by lymphatic cells. Space between trabeculae was increased, they were significantly narrowed. Lymphocytes were replaced by leukemic cells.

The splenic follicles were indistinguishable since the red pulp was filled with leukemic cells. Due to the paucity of germinal centres, a great number of undeveloped cells were observed.

Leukemic cells were found in myocardial fibres. The latter were dystrophic, thereby BLV-associated diffuse myocarditis was suspected. Some of them were indistinct, the nuclei were reduced and hematoxylin-stained intensely blue, whilst the sarcoplasm was eosin-stained dark red.

In the alveolar capillary network, erythrocytes were arranged in 3-4 rows, which could indicate hyperemia.

The structure of the liver was blurred at sites of leukemic cells accumulation. Granular and fatty liver degeneration of the liver was seen. Hepatocytes were swollen, whereas sinusoids were narrowed. Hepatocyte nuclei were poorly stained and hardly distinguishable, thus suggesting karyolysis.

There were foci of leukemic proliferation in the intercellular substance of renal connective tissue. Epitheliocytes of collecting tubules were inflamed and wall-thickened, with lumen narrowed. Some of their nuclei were narrowed, cell borders were poorly visible, and many small grains were eosin-stained pink in the cytoplasm of the epitheliocytes. This may point to renal cortical necrosis and granular dystrophy which are often seen in organisms affected by leukaemia [14].

Leukemic cells were noted in the duodenum mucosa as well.

On the mucosal surface of the small intestine, we detected a mixed exudate of neutrophils and lymphocytes, consisting of desquamated epithelial cells, mucus, and sanies. Postovalova et al. [15] reported a similar histological picture of the colorectal tissue of mice with experimental acute ulcerative colitis accompanied by the development of immunodeficiency.

In the small intestine, epithelial cells of the capillaries and adventitial cells were enlarged, which could be caused by oedema or plasmorrhagia due to immunopathological reactions $[11,16]$.

Diffuse clusters of leukemic cells were found between some skeletal muscle fibres along with their volume decreased.

With regard to the brain, we revealed diapedesis, pericellular oedema, and dystrophic changes in brain neurons, which are secondary manifestations of leukaemia in the brain [17].

Although our findings were sometimes difficult to interpret, these results confirm the evidence for the multicentric distribution of EBL in adult cattle [18]. 
To our knowledge, the current study is the first to report the description of microstructural changes involving lymph node trabeculae and myocardial fibres from leukemic cows. We have not found any published scientific data that would allow us to interpret the histological picture of these tissues, so we can only speculate that it stems from degenerative processes caused by BLV.

\section{Conclusion}

It can be concluded that our work provides new knowledge on histopathological features of EBL, which potentially may bring new insights into the disease mechanisms. Nevertheless, further studies using more cows should be conducted to clarify whether these alterations extend beyond the herd members.

The authors kindly acknowledge Sergey Sergeevich Kozhevnikov for English language editing.

\section{References}

1. A.A. Shabeykin, A.M. Gulyukin, T.V. Stepanova, N.G. Kozyreva, L.A. Ivanova, IOP Conf. Ser., 315, 042036 (2019)

2. S.N. Takeshima, Y. Kitamura-Muramatsu, Y. Yuan, M. Polat, S. Saito, Y. Aida, Arch. Virol., 160, 1325-1332 (2015)

3. A. De Brogniez, J. Mast, L. Willems, Viruses, 8, 88 (2016)

4. J.S. Lawson, B. Salmons, W.K. Glenn, Front. Oncol., 8, 1-18 (2018)

5. R.G. Olsen, S. Krakowka, J.R. Blakeslee, Comparative Pathobiology of Viral Diseases (CRC Press, Boca Raton, USA, 2019)

6. U. Braun, Vet. Clin. Food Anim. Pract., 32, 55-83 (2016)

7. M.G. Maxie (ed.), Jubb, Kennedy, and Palmer's Pathology of Domestic Animals (Elsevier, St. Louis, MO, 2016)

8. C.H. De Oliveira, J.D. Barbosa, K.A. Damasceno, G.D. Cassali, C.M. Oliveira, R.C. Leite, J.K. Reis, BMC Vet. Res., 12, 238 (2016)

9. P.D. Constable, K.W. Hinchcliff, S.H. Done, W. Grünberg, Veterinary medicine: $a$ textbook of the diseases of cattle, horses, sheep, pigs and goats (Elsevier Health Sciences, London, 2016)

10. EFSA Panel on Animal Health and Welfare (AHAW). Enzootic bovine leukosis, EFSA Journal, 13, 4188 (2015)

11. M.D. McGavin, J.F. Zachary, Pathologic basis of veterinary disease (Elsevier, St Louis, MO, 2017)

12. I.M. Donnik, A.S. Krivonogova, A.G. Isaeva, A.G. Koshchaev, O.P. Neverova, O.A. Bykova, Int. J. Green Pharm., 11, S620-S625 (2017)

13. J. McCune, F. Yuuka, CCAMLR Sci., 25, 273-278 (2018)

14. I.N. Gromov, E.I. Bol'shakova, I.V. Klimenkova, A.V. Svyatkovskij, P.S. Ryabcev, F. Alaraji, A.A. Svyatkovskij, M.M. Bykovskaya, Pathomorphological diagnosis of mycotoxicosis in poultry (Vitebsk State Academy of Veterinary Medicine, Vitebsk, 2016)

15. E.A. Postovalova, O.V. Makarova, Yu. Gao, Immunologiya, 37, 170-175 (2016)

16. I.R. Israilovich, T.K. Ziyaevich, E.E. Abdukhalimovich, Eur. Sci. Rev., 99-102 (2016) 
17. S.A. Kembhavi, S. Somvanshi, S. Banavali, P. Kurkure, B. Arora, Indian J. Radiol. Imaging, 22, 98 (2012)

18. L.S. Mello, W. Panziera, M.B. Bandinelli, L. Sonne, D. Driemeier, S.P. Pavarini, Pesq. Vet. Bras., 39, 32-39 (2019) 